\title{
Deuterium Link:
}

\section{From Interstellar Medium and Chemical Evolution to Cosmology and Structure Formation}

\author{
Tijana Prodanović ${ }^{* \dagger}$ \\ Department of Physics, University of Novi Sad, Trg Dositeja Obradovića 4, 21000 Novi Sad, \\ Serbia \\ E-mail: prodanvc@df.uns.ac.rs
}

\section{Gary Steigman}

Departments of Physics and Astronomy, Ohio State University 191 W. Woodruff Ave., Columbus OH 43210-1117, USA

E-mail: steigman@mps.ohio-state.edu

\section{Brian D. Fields}

Departments of Astronomy and of Physics, University of Illinois, Urbana, IL 61801, USA

E-mail: bdfields@illinois.edu

\begin{abstract}
Deuterium has a special place in cosmology, nuclear astrophysics, and galactic chemical evolution, because of its unique property that it is only created in the big bang nucleosynthesis while all other processes result in its net destruction. However, a large scatter found in the interstellar medium (ISM) deuterium abundance measurements indicates that deuterium might be preferentially depleted onto dust grains, which complicates the use of deuterium as a probe of galactic chemical evolution (GCE) models. We have applied a model-independent, statistical Bayesian method and determined the true, undepleted ISM D abundance. Having found the ISM D abundance, one can identify the successful GCE models, which can then be used to learn about nucleosynthesis in the ISM, but can also be placed in cosmological context to learn about the infall rates of the primordial gas to our Galaxy, which bares implications for models of galaxy formation. Here we present our results and their implications for discriminating between different GCE models, for our understanding of the nature and physics of interstellar dust grains, as well as implications for cosmological evolution.
\end{abstract}

11th Symposium on Nuclei in the Cosmos, NIC XI

July 19-23, 2010

Heidelberg, Germany

\footnotetext{
* Speaker.

$\dagger$ The work of TP is supported in part by the Provincial Secretariat for Science and Technological Development, and by the Ministry of Science of the Republic of Serbia under project 141002B. The research of GS is supported at The Ohio State University by a grant from the US Department of Energy.
} 


\section{Introduction}

Deuterium is only created in Big Bang [1] while all other processes destroy it [2,3]. This unique property makes $\mathrm{D}$ a powerful tool in cosmology as a cosmic baryometer - measurements of $\mathrm{D}$ abundance $y_{\mathrm{D}} \equiv(\mathrm{D} / \mathrm{H}) \times 10^{-5}$ in high redshift systems reveal a (close) to primordial $\mathrm{D}$ abundance, which is then used to set the baryon-to-photon ratio and predict other primordial abundances. Recent great agreement between primordial D abundance inferred from Wilkinson Microwave Anisotropy Probe measurements [4] and high-redshift low-metallicity observations [5] makes deuterium a BBN success story. The primordial D abundance is now adopted to be [6] $y_{\mathrm{D}_{\mathrm{p}}}=2.82_{-0.19}^{+0.20}$.

Of all the processes that destroy it, deuterium is most efficiently destroyed through stellar processing so that its abundance decreases monotonically from high to low redshift. This property makes deuterium also a powerful tool for GCR models as it thus probes the virgin fraction of the ISM - a fraction of gas that has never been processed in stars. However, recent FUSE measurements of deuterium abundance in the local ISM revel large variations [7, 8] - by a factor of 4 over different lines of sight (LOS) $0.5 \leq y_{\mathrm{D}_{\text {ISM }}} \leq 2.2$, which poses a problem for many, otherwise successful GCE models $[9,10]$. A solution has been proposed in the form of preferential deuterium depletion onto dust grains relative to hydrogen $[11,12]$. If this is the case, then a significant fraction of D abundance could be hidden in the dust, while the measured gas phase value is only a lower limit to the true, total ISM D abundance. Analyzing the most recent FUSE measurements, Linsky et al. 2006 [13] have revised a current estimate of the true, undepleted ISM D abundance and found it to be $y_{\mathrm{D}_{\text {ISM-dust }}} \geq 2.31 \pm 0.24$ which is at the $82 \%$ level of the primordial value! This result was based on the 5 LOS (of 46 available) which have the highest $\mathrm{D}$ abundances, and are thus, by assumption, least likely to suffer from depletion onto dust. Since D is destroyed through stellar cycling, such high present ISM abundance would require an astration factor of $f_{\mathrm{D}} \equiv y_{\mathrm{D}_{\mathrm{p}}} / y_{\mathrm{D}_{\text {ISM }}} \leq 1.22 \pm 0.15$ which is inconsistent with most GCE models that require $1.4 \leq f_{\mathrm{D}} \leq 1.8$. Moreover, if dust does hide large fraction of deuterium so that the true present ISM D abundance is close to primordial, should high-redshift system measurements also be corrected for the dust depletion?

\section{Galactic Chemical Evolution Models and Infall}

If so high [13], the ISM D abundance would imply that $80 \%$ of the initial interstellar gas has never been processed through stars. To account for this, most of the GCE models would require significant infall of (close to) pristine material which would bring fresh supply of D-rich gas in the ISM.

To test how much infall would be needed, Prodanović \& Fields 2008 [10] have constructed a simple and instructive GCE model, where the infall rate was assumed to be proportional to the starformation rate, with a proportionality constant $\alpha$. The predictions of this model were constrained by two observables: ISM-to-primordial deuterium mass fraction $D_{\mathrm{ISM}} / D_{\mathrm{p}}$, and present day gas fraction $w$. The results of the model directly depend on the assumed return fraction $R$ - a fraction of the initial stellar mass that is returned to the ISM (follows from an assumed initial mass function), and are presented on Figure 1. We see that ISM D abundance proposed by Linsky et al. 2006 [13] requires large infall rates that would have to almost completely balance star-formation rate 


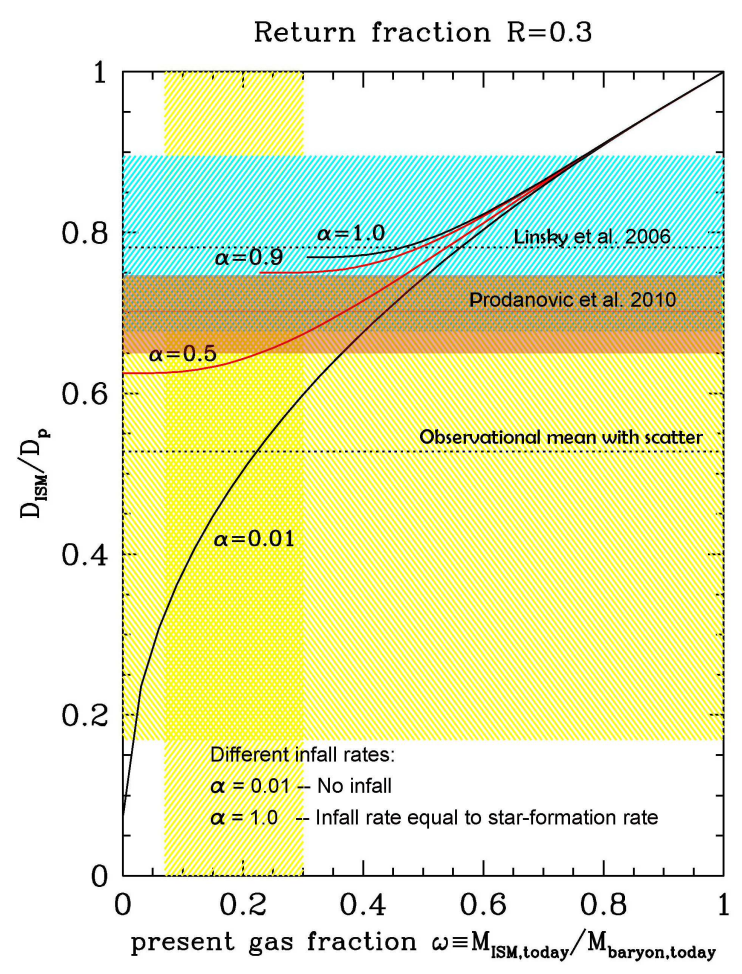

Figure 1: Presented are the results of Prodanović \& Fields 2008 [10]: the ratio of the total present-day to primordial deuterium mass fraction $D_{\mathrm{ISM}} / D_{\mathrm{p}}$ as a function of the present gas mass fraction $w$. Shaded areas represent observations, while solid lines are model results for different assumed infall parameter $\alpha$. Blue band reflects Linsky et al. 2006 [13] ISM D abundance, horizontal yellow band reflects measured D variations in the ISM, while the red band reflects the most recent prediction of the true, undepleted ISM D abundance [14]. The adopted primordial D abundance is that of [16].

with proportionality constant $0.5 \leq \alpha \leq 1.0$, in order to also account for the observed gas fraction, which is still in tension with most of the modern, successful GCE models.

\section{Determining the True ISM Deuterium Abundance: A Bayesian Approach}

Is the true ISM deuterium abundance really so high? To explore this, in [14] we took a fully statistical approach trying to make as little assumptions as possible. Following the work of Hogan et al. 1997 [17], we applied a model-independent Bayesian analysis on all available LOS, were the only assumption was that (dust) depletion may be possible. The goal is to find a two-parameter maximum likelihood function that best fits the available data. The output two parameters are $y_{\mathrm{D} \text {,max }}$ - maximum undepleted deuterium abundance consistent with the data, which is a lower limit to the true ISM D abundance $y_{\mathrm{D}, \max } \leq y_{\mathrm{D}, \mathrm{ISM}}$, and a depletion parameter $w=y_{\mathrm{D}, \max }-y_{\mathrm{D}, \min }$. Since the probability of some level of depletion along a given LOS is unknown, we also must adopt some form of a depletion distribution. We have tested 5 different depletion distributions: 1) top-hat bias , where all levels of depletion are equally probable, 2) negative bias, which favors large depletion, 3) positive bias, which favors low depletion, 4) M-shaped bias, where both high and low depletion 


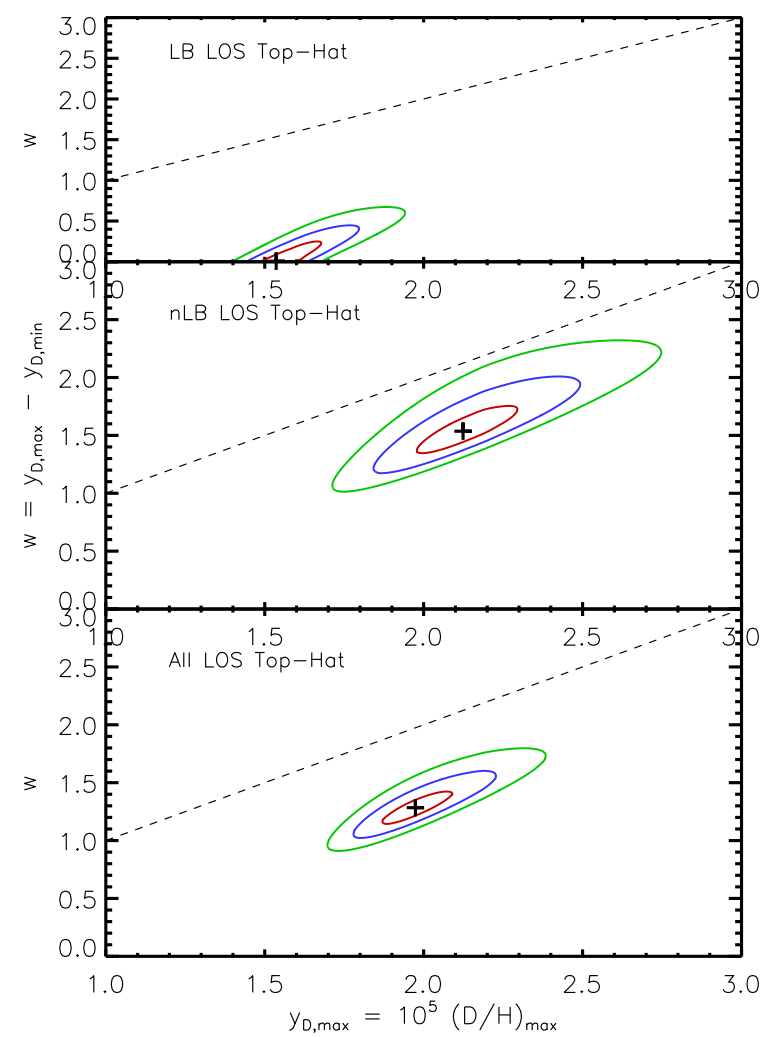

Figure 2: Likelihood contours $(68 \%, 95 \%, 99 \%)$ in the $y_{\mathrm{D}, \max }-\mathrm{w}$ plane for the 21 Local Bubble LOS (top panel), the 28 non-Local Bubble LOS (middle panel) and all 49 LOS (bottom panel) using the top-hat depletion distribution.

levels are equally favorable while moderate depletion levels are suppressed, 5) $\Lambda$-shaped bias, which strongly favors moderate depletion levels.

For each adopted depletion distribution we have separately completed a Bayesian analysis of the 49 available LOS D abundance measurements from [13] , and determined the combination of the two parameters values $y_{\mathrm{D}, \max }$ and $w$ that results in the largest maximum likelihood. Looking at the full data set and D abundances along those 49 LOS, it is easy to notice that there are two subsets of data - LOS that are within the Local Bubble (LB) where their D abundances show very little scatter and LOS that are outside of the Local Bubble (nLB) that show large scatter in D abundance measurements [13]. Thus, we have also applied our Bayesian analysis to these two subsets of data separately (Figure 2).

We have found that bayesian analysis done on all of the available ISM D measurements has the largest maximum likelihood value for a top-hat depletion distribution, where the best estimate of the true, undepleted ISM D abundance is [14]

$$
\left(\frac{D}{H}\right)_{\mathrm{ISM}} \geq\left(\frac{D}{H}\right)_{\max }=(2.0 \pm 0.1) \times 10^{-5}
$$

This new ISM D abundance is now consistent with some of the successful GCR models, as 
it requires a lower astration factor $f_{\mathrm{D}} \leq 1.4 \pm 0.1$. In terms of the gas mass observations and the required infall rate, Figure 1 shows that this new ISM D abundance of Prodanović et al. 2010 [14] requires somewhat lower infall rates with infall parameter now $0.4 \leq \alpha \leq 0.9$.

\section{Conclusion}

It was proposed that observed large variations of the ISM deuterium abundance are due to deuterium depletion onto dust grains preferentially relative to hydrogen. In the light of this hypothesis some of the new estimates of the true, undepleted ISM D abundance yield values that are close to primordial [13], which conflicts with many of the successful GCE models. Our Bayesian statistical approach yields somewhat lower value $(\mathrm{D} / \mathrm{H})_{\mathrm{ISM}}=20 \mathrm{ppm}$ which relieves the tension with GCE models [14] but still requires significant infall rates of close-to-pristine material [10]. Still, some questions remain - Why is the Local Bubble deuterium abundance so uniform and depleted from the ISM value, while iron depletion shows significant scatter? Is ISM D abundance really so high or some of the high measured values reflect recent unmixed infall of pristine gas? Should highredshift observations of deuterium also be corrected for the potential depletion onto dust? These questions will be further analyzed in future work.

\section{References}

[1] A. M. Boesgaard, G. Steigman, Ann. Rev. Astron. Astrophys., 23, 319 (1985).

[2] R. I. Epstein, J. M. Lattimer, D. N. Schramm, Nature, 263, 198 (1976).

[3] T. Prodanović, B. D. Fields, ApJ, 597, 48 (2003).

[4] D. N. Spergel, et al., ApJS, 170, 377 (2007).

[5] M. Pettini, B. J. Zych, M. T. Murphy, A. Lewis, C. C. Steidel, MNRAS, 391, 1499 (2008).

[6] R. H. Cyburt, B. D. Fields, K. A. Olive, JCAP, 11, 12 (2008).

[7] E. B. Jenkins, T. M. Tripp, P. R. Woźniak, U. J. Sofia, G. Sonneborn, ApJ, 520, 182 (1999).

[8] C. G. Hoopes, K. R. Sembach, G. Hébrard, H. W. Moos, D. C. Knauth, ApJ, 586, 1094 (2003).

[9] G. Steigman, D. Romano, M. Tosi, 2007, MNRAS, 378, 576 (2007).

[10] T. Prodanović, B. D. Fields, JCAP, 9, 3 (2008).

[11] M. Jura, Advances in Ultraviolet Astronomy, Kondo Y., Mead J., Chapman R. D., eds., NASA, Washington, p. 54 (1982).

[12] B. T. Draine, Astrophysics in the Far Ultraviolet: Five Years of Discovery with FUSE, 348, 58 (2006).

[13] J. L. Linsky, et al., ApJ, 647, 1106 (2006).

[14] T. Prodanović, G. Steigman, B. D. Fields, MNRAS, 406, 1108 (2010).

[16] R. H. Cyburt, B. D. Fields, K. A. Olive, Phys. Lett. B, 567, 227 (2003).

[17] C. J. Hogan, K. A. Olive, S. T. Scully, ApJL, 489, L119 (1997). 